\title{
Health-related quality of life and health utility among patients with diabetes in Zabol, Southeast Iran
}

\author{
Seyed Pouria Hedayati ${ }^{1}$, Hassan Haghparast-Bidgoli², Aliasghar A. Kiadaliri', Fatemeh Mohabati ${ }^{1}$
}

\author{
'Department of Health Management and Economics, School of Public Health, Tehran University of Medical Sciences, Tehran, Iran. \\ ${ }^{2}$ Institute for Global Health, University College London, London, UK \\ ${ }^{3}$ Department of Clinical Sciences Lund, Lund University, Faculty of Medicine, Orthopaedics, Clinical Epidemiology Unit, Lund, Sweden. \\ Corresponding Author: Fatemeh Mohabati (E-mail: mohabatif@gmail.com) \\ (Submitted: 16 January 2020 - Revised version received: 10 February 2020 - Accepted: 14 March 2020 - Published online: 26 April 2020)
}

\begin{abstract}
Objectives The current study aimed to assess the factors associated with health-related quality of life (HRQoL) and health utility (HU) among patients with diabetes in Zabol, Southeast Iran.

Methods Among patients referred to Zabol city diabetes clinic, a total of 213 consecutive patients 18 years and older consent to participate in the study in 2015. The Persian version of EuroQol-5D-3L (EQ-5D-3L) using the UK preference weights was applied to derive HU. Logistic regression and ordinary least squares were used for data analysis. The STATA version 13 (StataCorp LP, College Station, TX, USA) was used for statistical analysis.

Results The highest and lowest proportions of "some or extreme problems" were seen in pain/discomfort (86.6\%) and self-care (27.8\%) dimensions of the EQ-5D-3L, respectively. About $33 \%$ of women and $14 \%$ of men rated their health worse than death ( $p=0.002$ ). The mean EQ-5D-3L index score and visual analogue scale were 0.37 (95\% Cl: 0.31-0.42) and 51.6 (95\% Cl: 48.7-54.5), respectively. Older age at diagnosis, longer duration of diabetes, lower education, and history of macrovascular complications were associated with lower HRQoL and $\mathrm{HU}$.

Conclusion This study highlights the importance of education and diabetes-related complications in HRQoL/HU of diabetes people. The findings suggest that urgent interventions are required to improve HRQoL/HU of diabetes patients in Zabol. Moreover, our results provide inputs for future economic evaluation studies among diabetes patients with similar socioeconomic status in Iran.

Keywords Diabetes, Health-related quality of life, Health utility, EQ-5D-3L, Iran.
\end{abstract}

\section{Introduction}

Diabetes mellitus is a chronic disease with a rising prevalence globally. The number of diabetic patients in 2011 was estimated to be around 366 million worldwide, and it is predicted that this figure will rise to more than $50 \%$ by 2030 . A prevalence of $12.5 \%$ was estimated among people aged $20-79$ years in Middle East and North Africa region. ${ }^{1}$ For the Iranian population, prevalence has increased from $7.7 \%$ in 2005 to $10.3 \%$ in $2016 .^{2-4}$

Diabetes elevates risk of a number of macro- and microvascular complications. ${ }^{5,6}$ A pooled analysis of 8.49 million person-years at risk, from 102 prospective studies, indicated that hazard ratios (HRs) of coronary heart disease, ischemic stroke and hemorrhagic stroke were 2, 2.27 and 1.56 for diabetic compared with non-diabetic peoples, respectively. ${ }^{7}$ A population-based study in Iran found that HRs of having cardiovascular disease (CVD) were 3.30 and 1.90 for women and men with diabetes compared with people without diabetes, respectively. ${ }^{8}$ The association between diabetes and complications causes both a shorter life expectancy ${ }^{9}, 10$ and a poorer health-related quality of life (HRQoL) among the diabetic patients compared to those without diabetes. ${ }^{11}$ Similarly, diabetic patients with diabetes-related complications have lower HRQoL compared to diabetic patients without any complication. ${ }^{12-15}$

There has been a growing interest in assessing HRQoL in patients with diabetes in Iran over the recent years. These studies have reported a negative impact of diabetes-related complications on HRQoL. ${ }^{16-18}$ HRQoL is a multidimensional concept that includes domains related to physical, mental, emotional, and social functioning. It goes beyond direct measures of population health, life expectancy, and causes of death, and focuses on the impact health status has on quality of life, for example, for diabetic patients. ${ }^{19}$ While, HRQoL is an important outcome in evaluating the effects of different health states and assessing the effectiveness of various interventions, it is patients' health utilities (HUs) which are the main interest in the context of economic evaluation..$^{20}$ Some approaches to economic evaluation in the health sector are: Cost of Illness Studies, Cost Benefit Analysis, Cost Effective Analysis (CEA), Cost Utility Analysis. In Cost Utility Analysis, an extension of CEA but enables comparisons of different treatments with quite different outcomes. This is especially when interventions cause differences in the quantity (survival) and quality of life. It does this through combining these in the common metric of the Quality Adjusted Life Year (QALY). The costs data for achieving a QALY mean that different interventions for the same illness/conditions can be compared as can interventions for other conditions. It can be used to assess whether drugs should be listed on PBS and to guide resource allocation decisions HUs are used to calculate QALYs, as a common outcome measure in cost-utility analyses. ${ }^{21} \mathrm{HU}$ is a measure of individual's preferences for different health outcomes. It is a cardinal value, usually between 0 and 1 , covering different health states from the worst to perfect health. ${ }^{20}$ These HU values are generally combined with survival estimates to generate QALY (e.g., 2 years with a $\mathrm{HU}$ value of 0.7 and 2 years with a $\mathrm{HU}$ value of 0.5 generate a QALY value of 2.9 for these 5years survival). ${ }^{20}$ There are two main approaches to elicit the patients' HU: direct method such as standard gamble and time trade-off, and indirect method using preference-based measures such as 
EuroQol-5D (EQ-5D) ${ }^{22}$ and SF-6D. ${ }^{23-25}$ Among generic instruments, EQ-5D, WHOQOL, Health Utility Index, Quality of Well-Being, and SF-36 have been used internationally. We selected EQ-5D because, among these instruments, it has the advantage of being able to calculate a single comprehensive scalar unit of values that can be compared among diseases and used for economic evaluation. EQ-5D is a preference-based HRQL questionnaire that was developed in Europe. ${ }^{26}$ We measured HRQL in patients with diabetes using EQ-5D, one of the preference-based measures among HRQL instruments that enable calculation of the utility value. ${ }^{27}$ To our knowledge, only one previous study measured $\mathrm{HU}$ among patients with type 2 diabetes using the EQ-5D in Iran (28). They found that while CVD and nephropathy had a negative impact on HU, there was positive association between having retinopathy and HU. ${ }^{28}$

The primary aim of the current study was to assess demographic, socioeconomic, and clinical correlates of HRQoL/ HU among patients with diabetes in a deprived area of Iran. In addition, since event-specific effects on HU are more useful in conducting economic evaluation studies, our secondary aim was to evaluate the effect of macrovascular complications including myocardial infarction (MI), coronary heart disease and stroke as separate events, not pooled as CVD, on patients HU.

\section{Methods}

\section{Design and Subjects}

A cross-sectional study was conducted among patients referred to a hospital-based diabetes clinic in Zabol in 2015, 213 of 254 consecutive patients (with either type 1 or type 2 diabetes) who visited the diabetes clinic met the inclusion criteria for the current study: (a) age 18 years or older, (b) consent to participate in the study. These were patients with confirmed diabetes by physicians in the clinic. This is the only diabetes clinic in Zabol. The study was approved by the Zabol University of Medical Sciences' ethical committee.

\section{Explanatory Variables}

A questionnaire was designed by the research team to collect the data on the variables required for the current study. The main variables included year of birth, year of diagnosis of diabetes, gender, resident place, household income, employment status, years of education, weight $(\mathrm{kg})$, height $(\mathrm{cm})$, and history of self-reported doctor's diagnosed macrovascular diabetes-related complications. Patients were asked if they visited a physician for any of the following complications during last year: stroke, MI, and coronary heart disease. A categorical variable using three quintiles of household income was used as the measure of socioeconomic status in this analysis.

\section{Health-related quality of life and health utility assessment}

The Persian version of EQ-5D-3L was used to assess HRQoL/ HU in the study. The EQ-5D-3L is simple to use and have shown good performance among people with diabetes in previous studies. ${ }^{29,30}$ The EQ-5D-3L is a multiattribute preference-based instrument which constitute of five dimensions: mobility, self-care, usual activities, pain/discomfort, and anxiety/depression. Each dimension has three levels: no problems, some problems, and extreme problems, ${ }^{22}$ which result in
$243^{35}$ potential health states. Responses to these dimensions are weighted based on the preference elicited from a sample of general population to compute an index score. The EQ-5D-3L index score ranges from less than 0 (negative values) for health states worse than death to 1 for full health. The EQ-5D-3L questionnaire also contains a visual analog scale (VAS) tool which entails respondent rates his/her current health state on a scale from 0 , the worst imaginable health state, to 100 , the best imaginable health state. In the current study, due to lack of preference weights for the Iranian population, the value sets for UK population ${ }^{31}$ was used. Patients responded to the EQ-5D-3L questionnaire through a face-to-face interview, conducted by a trained interviewer. This questionnaire has been translated by the EuroQol Group into various languages, and for this study, after filling out a form about the current study method on the group's website, the translated and validated version was sent to the participants. The reliability and validity of the EQ-5D have been well-documented in different contexts for different diseases. ${ }^{32-34}$

\section{Data Analysis}

The continuous variables are shown as mean and standard deviation and the categorical as percentages. Responses to the EQ-5D-3L questions were merged for all five dimensions and a binary outcome as "no problem" or "some or extreme problem" was created. Then, $\chi^{2}$ and Logistic regression were used to assess the associations between the explanatory variables and these binary variables. The STATA version 13 (Stata Corp LP, College Station, TX, USA) was used for statistical analysis.

The EQ-5D-3L index score and VAS were analyzed using $t$-test, analysis of variance (ANOVA) and ordinary least squares (OLS). Due to skewed nature of the EQ-5D-3L scores, several methods have been used in the literature to analyze the scores. ${ }^{35-37}$ We chose the OLS with robust standard errors in the current study for two main reasons: first, only $5 \%$ the patients reported no problem in any dimensions of the EQ-5D-3L (i.e., an index score $=1.0$ ) and $1 \%$ reported a VAS score of 100 . It is shown that in this situation OLS works as well as other methods. ${ }^{36}$ Second, when HU is the main interest of analysis, as in the current study, the OLS with robust standard errors is a valid approach. ${ }^{37}$

As education level is highly associated with employment and income, we excluded income and employment status from multivariate analysis to avoid any mediation bias. Years of education was categorized in two level: 8 years and less, and more than 8 years. Three patients with missing value on the year of diagnosis were excluded from the analysis. The design variables and residual plots were used to check the linearity of the continuous variables and continuous covariates were treated as mean-centered values. The STATA version 13 (Stata Corp LP, College Station, TX, USA) was used for statistical analysis. Participants were asked to participate on a voluntary basis. They were informed about the study objectives, procedures, risks, benefits, alternatives, their rights, and data anonymity and confidentiality. This information was included in the informed consent form signed by the participants.

\section{Results}

The mean (SD) age at diagnosis and duration of diabetes were $39.86( \pm 13.36)$ years and $9.73( \pm 7.10)$ years, respectively. Sixty three percent of the sample were male and $31 \%$ had a BMI $\geq$ 
30 (Table 1). The macrovascular complications were more common among men. "Some or extreme problems" in pain/discomfort dimension has the highest prevalence, with $86.6 \%$, followed by anxiety/depression ( $84.5 \%)$. In total, $33.3 \%$ of women and $14.1 \%$ of men rated their health worse than death (i.e., EQ-5D-3L score $<0, P=0.002)$. The mean (95\% CI) EQ-5D-3L index score and VAS scale were $0.37(0.31-0.42)$ and 51.6 (48.7$54.5)$, respectively. The Spearman rank correlation between the EQ-5D index score and VAS scale was $0.65(P<0.001)$.

The univariate analysis showed that men and people diagnosed at age older than 25 years had lower problems on the EQ-5D-3L dimensions (Table 2). Better socioeconomic status was generally associated with lower frequency of the problems. The patients with a history of macrovascular events generally suffered from more problems, but this was not statistically significant for pain/discomfort and anxiety/ depression dimensions. Patients who received combination of insulin and oral hypoglycemic agents (OHA) had statistically significantly more difficulties in doing their usual daily activities.

Table 1. Demographic, socioeconomic and clinical characteristics of the sample, stratified by sex $(N=213)$

\begin{tabular}{|c|c|c|}
\hline Variable & Men & Women \\
\hline $\mathrm{N}$ & 78 & 135 \\
\hline Age at diagnosis, years & $39.87 \pm 15.35$ & $39.86 \pm 12.12$ \\
\hline Diabetes duration, years & $10.70 \pm 8.33$ & $9.17 \pm 6.24$ \\
\hline BMI & $26.08 \pm 4.50$ & $28.85 \pm 4.57$ \\
\hline \multicolumn{3}{|l|}{ Treatment } \\
\hline ОНА (\%) & 57.69 & 48.15 \\
\hline Insulin \pm OHA (\%) & 42.31 & 51.85 \\
\hline History of MI (\%) & 7.69 & 11.11 \\
\hline History of coronary heart disease (\%) & 15.38 & 14.81 \\
\hline History of stroke (\%) & 5.13 & 12.59 \\
\hline \multicolumn{3}{|l|}{ Household income } \\
\hline Low (\%) & 38.46 & 47.41 \\
\hline Middle (\%) & 26.92 & 31.11 \\
\hline High (\%) & 34.62 & 21.48 \\
\hline \multicolumn{3}{|l|}{ Employment } \\
\hline Unemployed / housekeeper (\%) & 12.82 & 90.37 \\
\hline Employed (\%) & 38.46 & 5.93 \\
\hline Retired (\%) & 48.72 & 3.70 \\
\hline \multicolumn{3}{|l|}{ Education } \\
\hline No education (\%) & 14.10 & 50.37 \\
\hline $1-8$ years $(\%)$ & 33.33 & 34.07 \\
\hline 9-12 years $(\%)$ & 37.18 & 13.33 \\
\hline$>12$ year (\%) & 15.38 & 2.22 \\
\hline
\end{tabular}

BMl: body mass index, OHA: oral hypoglycaemic agents, MI: myocardial infarct.
The results of logistic regression showed no gender difference in suffering from problems in the EQ-5D-3L dimensions (Table 3). Except for self-care, there was no statistically significant association between age at diagnosis and having problems in any EQ-5D-3L dimensions. Longer duration of diabetes and history of macrovascular complications were associated with higher odds of having problems. People with higher education had statistically significantly lower odds of having problems in all dimensions.

The results of OLS regression revealed that older age at diagnosis, longer duration of diabetes, lower education, and history of macrovascular complications were associated with lower EQ-5D-3L index scores (Table 4). A similar finding was observed when VAS scale was used as dependent variable, except for history of stroke which was no longer statistically significant.

\section{Discussion}

In order to support conducting economic evaluation of diabetes preventive or curative interventions in Iran, we have estimated HU scores for a range of factors, including demographic, socioeconomic, and clinical factors, among patients referred to a clinic in Southeast Iran. As one may expect, macrovascular complications' history was associated with lower HRQoL/HU among patients with diabetes. Among these complications, history of MI had the highest negative impact on EQ-5D-3L index score. In addition, higher education was associated with higher HRQoL/HU among patients with diabetes Similar to previous national and international studies, ${ }^{28,38-40}$ people with diabetes had more commonly problem on pain/discomfort and anxiety/depression dimensions of the EQ-5D-3L and had least problem in self-care. In addition, physician, nurses, and other caregivers should pay more attention on these dimensions. Our findings on the association between education, age at diagnosis, and duration of diabetes with HRQoL/HU were in line with previous national and international studies. ${ }^{13,30,41-43}$. These findings have important clinical and policy-making implications since identifying the most affected dimensions of HRQoL and its determinant can guide toward a better management of the disease and improving HRQoL in these patients.

We found no significant association between treatment modality and HRQoL/HU. This might be due to poor sensitivity of the EQ-5D-3L to treatment modality in diabetes context as has been previously shown. ${ }^{15,28,44}$ While the mean VAS score was closer to the value reported in the national survey of type 2 diabetes ( 51.5 vs. 56.8 ), the mean EQ-5D index score in the current study was significantly lower than the national survey ( 0.37 vs. 0.70$).^{28}$ There are several possible explanations for this disparity: first, our study included the patients who were referred to a clinic who might be potentially sicker than general diabetes population included in the national survey. Second, Zabol city located in Sistan \& Baluchestan province that is considered as one of the most deprived provinces in the country. This poor socioeconomic status not only can directly affect the patients' HRQoL/HU, but also influences the quality and access to care and treatment for patients in this city. Third, we included both type 1 and type 2 diabetes patients while in the national survey only type 2 diabetes patients were included. Patients with type 1 diabetes are diagnosed in younger age, therefore have 


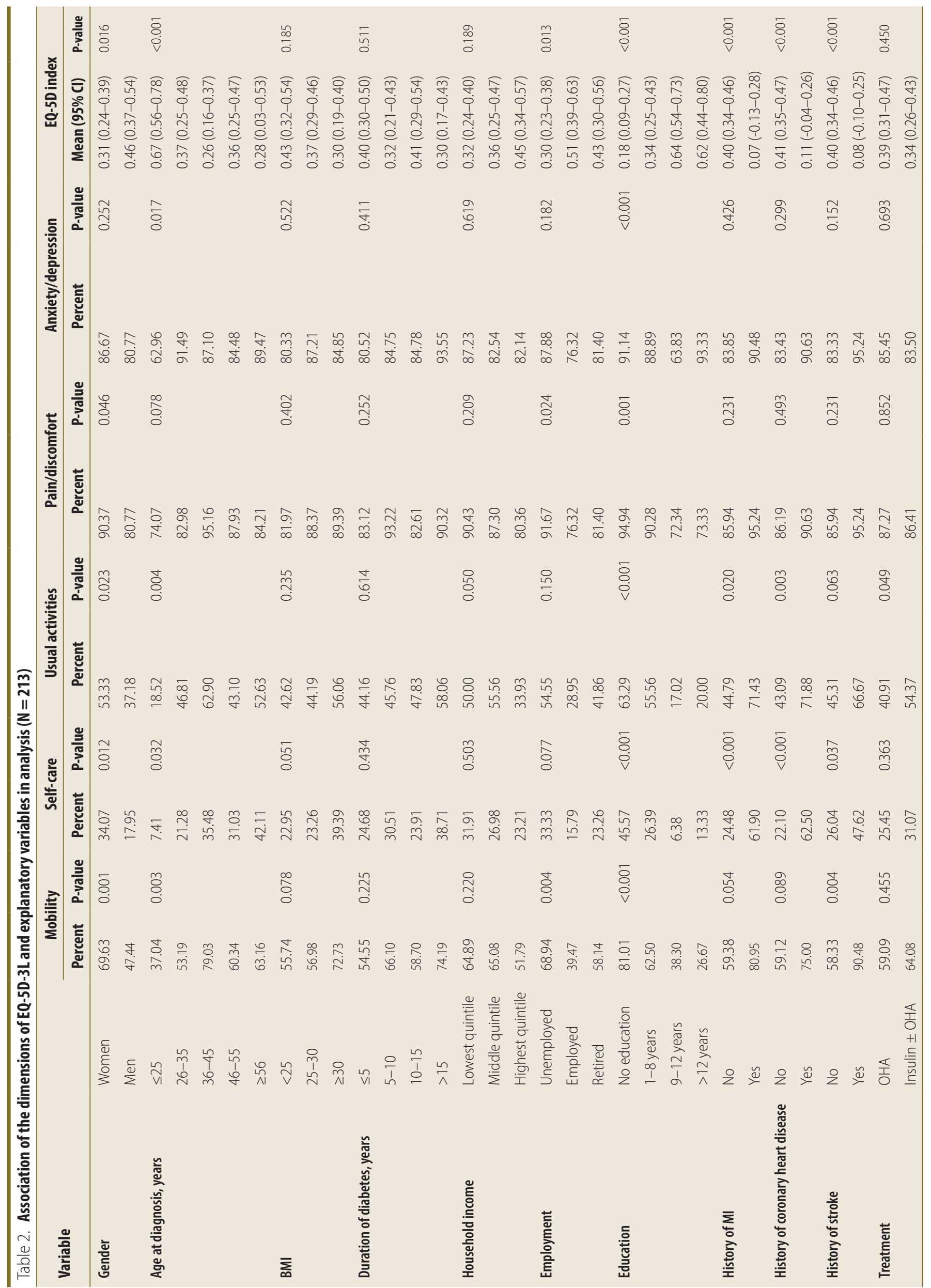




\begin{tabular}{|c|c|c|c|c|c|c|c|c|c|c|c|}
\hline \multirow[t]{2}{*}{ Variable } & & \multicolumn{2}{|c|}{ Mobility } & \multicolumn{2}{|c|}{ Self-care } & \multicolumn{2}{|c|}{ Usual activities } & \multicolumn{2}{|c|}{ Pain/discomfort } & \multicolumn{2}{|c|}{$\begin{array}{c}\text { Anxiety/ } \\
\text { depression }\end{array}$} \\
\hline & & OR & P-value & OR & P-value & $\mathrm{OR}$ & P-value & OR & P-value & OR & P-value \\
\hline Men & & 0.58 & 0.147 & 0.57 & 0.217 & 0.96 & 0.913 & 0.68 & 0.398 & 0.92 & 0.854 \\
\hline Age at diagnosis & & 1.02 & 0.183 & 1.05 & 0.007 & 1.02 & 0.159 & 1.02 & 0.396 & 1.01 & 0.549 \\
\hline Diabetes duration & & 1.08 & 0.004 & 1.10 & 0.003 & 1.05 & 0.058 & 1.04 & 0.189 & 1.05 & 0.095 \\
\hline Body mass index & & 1.07 & 0.053 & 1.07 & 0.140 & 1.04 & 0.233 & 1.01 & 0.824 & 0.99 & 0.833 \\
\hline \multirow[t]{2}{*}{ Education } & $\leq 8$ years (ref) & 1.00 & - & 1.00 & - & 1.00 & - & 1.00 & - & 1.00 & - \\
\hline & $>8$ years & 0.36 & 0.009 & 0.23 & 0.013 & 0.18 & $<0.001$ & 0.30 & 0.013 & 0.32 & 0.015 \\
\hline \multirow[t]{2}{*}{ Treatment } & $\mathrm{OHA}$ (ref) & 1.00 & - & 1.00 & - & 1.00 & - & 1.00 & - & 1.00 & - \\
\hline & Insulin $\pm \mathrm{OHA}$ & 1.14 & 0.716 & 1.08 & 0.846 & 1.73 & 0.103 & 0.85 & 0.738 & 0.71 & 0.458 \\
\hline History of MI & & 2.84 & 0.090 & 6.64 & 0.003 & 3.20 & 0.043 & 2.77 & 0.318 & 1.58 & 0.549 \\
\hline History of coronary heart disease & & 1.87 & 0.193 & 7.00 & $<0.001$ & 3.80 & 0.003 & 1.25 & 0.717 & 1.55 & 0.526 \\
\hline History of stroke & & 4.63 & 0.042 & 1.35 & 0.619 & 1.36 & 0.589 & 1.58 & 0.646 & 2.50 & 0.338 \\
\hline
\end{tabular}

OR: odds ratio.

\begin{tabular}{|c|c|c|c|c|c|}
\hline \multirow{2}{*}{ Variable } & & \multicolumn{2}{|c|}{ EQ-5D index score } & \multicolumn{2}{|c|}{ Visual analog scale } \\
\hline & & Coefficient & P-value & Coefficient & P-value \\
\hline Men & & 0.005 & 0.927 & 1.005 & 0.756 \\
\hline Age at diagnosis & & -0.004 & 0.050 & -0.279 & 0.012 \\
\hline Diabetes duration & & -0.007 & 0.036 & -0.567 & 0.003 \\
\hline Body mass index & & -0.006 & 0.301 & -0.285 & 0.356 \\
\hline \multirow[t]{2}{*}{ Education } & $\leq 8$ years (ref) & 0.000 & - & 0.000 & - \\
\hline & $>8$ years & 0.291 & $<0.001$ & 11.498 & $<0.001$ \\
\hline \multirow[t]{2}{*}{ Treatment } & $\mathrm{OHA}$ (ref) & 0.000 & - & 0.000 & - \\
\hline & Insulin $\pm \mathrm{OHA}$ & -0.021 & 0.682 & -3.150 & 0.282 \\
\hline History of MI & & -0.295 & 0.002 & -12.099 & 0.011 \\
\hline History of coronary heart disease & & -0.250 & $<0.001$ & -8.536 & 0.026 \\
\hline History of stroke & & -0.188 & $<0.001$ & -1.425 & 0.688 \\
\hline Constant & & 0.376 & $<0.001$ & 52.035 & $<0.001$ \\
\hline
\end{tabular}

dx.doi.org/10.22317/jcms.v6i2.727

longer duration of diabetes and this might have caused lower HRQoL/HU in the current study. In addition, a previous study showed that diabetes-related complications have more negative impact on HRQoL among type 1 diabetes patients with younger age.

The range of HU decrement due to macrovascular complications was higher in our study than the estimates reported in previous studies. ${ }^{45}$ This may reflect either poor access to secondary health care or low quality of care for patients with diabetes in such deprived area. In addition, such differences imply that the cost-utility analyses using estimates from previous national study might not be generalizable to diabetes patients in this deprived area of the country.
The results of the current study should be interpreted in light of a number of limitations. First, the sampling method was non-random, and this negatively affects representativeness of the patients and limits generalizability of the results presented here. Second, the data were self-reported, with risk of potential recall bias and measurement errors that might bias the results. Third, we used the preference weights from the UK population to calculate the EQ-5D-3L index score. Due to intercultural differences in health state preferences, ${ }^{46-48}$ this might be problematic. Fourth, both type 1 and type 2 diabetes were included in the study, which may limit transparency and comparability of the results. Fifth, as this is a cross-sectional study, any causal inference from the results should be avoided. 


\section{Conclusion}

The current study has estimated HU scores for a range of demographic and clinical features of diabetic patients in a deprived area of Iran. The findings showed that older age at diagnosis, longer duration of diabetes, lower socioeconomic status, and history of macrovascular complications were associated with lower HRQoL/HU. The findings also showed that the mean EQ-5D-3L index score in the sample of diabetes patients in the current study was lower than the Iranian diabetes general population, implying that specific interventions should be implemented to improve HRQoL of patients in this area. Using these estimates in conducting cost-utility analyses can assist informed decisions by policy-makers in Iran. Assessing the effects of microvascular complications on HRQoL/HU and evaluating the changes of HRQoL/HU over time in a larger sample size are topics for future research.

\section{Acknowledgments}

It is acknowledged that the current study was approved by Zabol University of Medical Sciences the current study was not funded. The authors would like to thank the staff of the diabetes center in Zabol, Iran, for their great assistance in this project. The authors would like to thank the staff of the diabetes center in Zabol, Iran, for their great assistance in this project.

\section{Conflicts of interest}

The authors declare that there is no conflict of interests.

\section{References}

1. Whiting DR, Guariguata L, Weil C, Shaw J. IDF diabetes atlas: Gglobal estimates of the prevalence of diabetes for 2011 and 2030. Diabetes Res Clin Pract. 2011;94(3):311-321. doi:10.1016/j.diabres.2011.10.029

2. Esteghamati A, Gouya MM, Abbasi M, et al. Prevalence of diabetes and impaired fasting glucose in the adult population of Iran: National Survey of Risk Factors for Non-Communicable Diseases of Iran. Diab Care. 2008:31(1):96-98. doi:10.2337/dc07-0959

3. Esteghamati A, Meysamie A, Khalilzadeh O, et al. Third national Surveillance of Risk Factors of Non-Communicable Diseases (SuRFNCD-2007) in Iran: Methods and results on prevalence of diabetes, hypertension, obesity, central obesity, and dyslipidemia. BMC Public Health. 2009:9:167. doi:10.1186/1471-2458-9-167

4. World Health Organization - NCD Country Profiles (2016). Available from: http://www.who.int/diabetes/country-profiles/irn_en.pdf

5. McVeigh GE, Gibson W, Hamilton PK. Cardiovascular risk in the young type 1 diabetes population with a low 10-year, but high lifetime risk of cardiovascular disease. Diab Obes Metab. 2013;15(3):198-203. doi:10.1111/ dom.12013

6. Goeree R, Lim ME, Hopkins R, et al. Excess risk of mortality and complications associated with newly diagnosed cases of diabetes in Ontario, Canada. Can J Diab. 2009;33(2):93-104. doi:10.1016/S14992671(09)32006-7

7. Emerging Risk Factors Collaboration, Sarwar N, Gao P, Seshasai SR, et al. Diabetes mellitus, fasting blood glucose concentration, and risk of vascular disease: A collaborative meta-analysis of 102 prospective studies. Lancet. 2010;375(9733):2215-2222. doi:10.1016/S0140-6736(10)60484-9

8. Hadaegh F, Khalili D, Fahimfar N, Tohidi M, Eskandari F, Azizi F. Glucose intolerance and risk of cardiovascular disease in Iranian men and women: Results of the 7.6-year follow-up of the Tehran Lipid and Glucose Study (TLGS). J Endocrinol Invest. 2009;32(9):724-730. doi:10.3275/6399

9. Franco OH, Steyerberg EW, Hu FB, Mackenbach J, Nusselder W. Associations of diabetes mellitus with total life expectancy and life expectancy with and without cardiovascular disease. Arch Intern Med. 2007;167(11):1145-1151. doi:10.1001/archinte.167.11.1145
10. Morgan CL, Currie CJ, Peters JR. Relationship between diabetes and mortality: a population study using record linkage. Diab Care 2000;23(8):1103-1107.

11. Koopmanschap M, Board C-A. Coping with Type II diabetes: the patient's perspective. Diabetologia. 2002;45(7):S18-22. doi:10.1007/s00125-0020861-2

12. Fu AZ, Qiu Y, Radican L, Luo N. Marginal differences in health-related quality of life of diabetic patients with and without macrovascular comorbid conditions in the United States. Qual Life Res. 2011:20(6):825-832. doi:10.1007/s11136-010-9819-x

13. Zhang P, Brown MB, Bilik D, Ackermann RT, Li R, Herman WH. Health utility scores for people with type 2 diabetes in U.S. managed care health plans: results from Translating Research Into Action for Diabetes (TRIAD). Diab Care. 2012;35(11):2250-2256. doi:10.2337/dc11-2478

14. O'Reilly DJ, Xie F, Pullenayegum E, et al. Estimation of the impact of diabetes-related complications on health utilities for patients with type 2 diabetes in Ontario, Canada. Qual Life Res. 2011;20(6):939-943. doi:10.1007/ s11136-010-9828-9

15. Kiadaliri AA, Gerdtham UG, Eliasson B, Gudbjornsdottir S, Svensson AM, Carlsson KS. Health utilities of type 2 diabetes-related complications: A cross-sectional study in Sweden. Int J Environ Res Public Health. 2014:11(5):4939-4952. doi:10.3390/ijerph110504939

16. Ghanbari A, Yekta ZP, Roushan ZA, Lakeh NM. Assessment of factors affecting quality of life in diabetic patients in Iran. Public Health Nurs. 2005; 22(4):311-322. doi:10.1111/j.0737-1209.2005.220406.x

17. Sanjari M, Safari S, Shokoohi M, et al. A cross-sectional study in Kerman, Iran, on the effect of diabetic foot ulcer on health-related quality of life. Int J Low Extrem Wounds. 2011;10(4):200-206. doi:10.1177/1534734611428728

18. Kiadaliri AA, Najafi B, Mirmalek-Sani M. Quality of life in people with diabetes: a systematic review of studies in Iran. J Diab Metab Disord. 2013; 12(1):54. doi:10.1186/2251-6581-12-54

19. Ferrans CE. Definitions and conceptual models of quality of life. In: Lipscomb J, Gotay CC, Snyder C, (eds.) Outcomes assessment in cancer. Cambridge, England: Cambridge University; 2005. pp. 14-30.

20. Torrance GW. Utility approach to measuring health-related quality of life J Chronic Dis. 1987:40(6):593-603.

21. J. E. Sansoni "Instruments for economic evaluation: session 5", Managing and Measuring Health Outcomes, Menzies School of Health Research, Darwin, 8-11 March 2011.

22. Brooks R. EuroQol: the current state of play. Health Policy. 1996:37(1):53-72.

23. Brazier J, Roberts J, Deverill M. The estimation of a preference-based measure of health from the SF-36. J Health Econ. 2002; 21(2):271-292.

24. Hatswell A, Pennington B, Pericleous L, Rowen D, Lebmeier M, Lee D. Patient-reported utilities in advanced or metastatic melanoma, including analysis of utilities by time to death. J Health Quality of Life Outcomes 2014; $12: 14$.

25. Cruz L.N, Camey S.A, Hoffmann J.F, Rowen D, Brazier J.E. Fleck M. P, Polanczyk C.A. Estimating the SF6D value set for a population-based sample of Brazilians. JValue in Health. 2011;1(4): 108-14.

26. Sakamaki H, et al. Measurement of HRQL using EQ-5D in patients with type 2 diabetes mellitus in Japan. J Int Soc Pharmaco Econ Outcomes Res (ISPOR). 2006:9(1):47-53.

27. EuroQol Group. EuroQol — a new facility for the measurement of healthrelated quality of life. Health Policy 1990;16:199-208.

28. Javanbakht M, Abolhasani F, Mashayekhi A, Baradaran HR, Jahangiri noudeh Y. Health related quality of life in patients with type 2 diabetes mellitus in Iran: A national survey. PLoS One. 2012;7(8):e44526. doi:10.1371/journal. pone.0044526

29. Glasziou P, Alexander J, Beller E, Clarke P, Group AC. Which health-related quality of life score? A comparison of alternative utility measures in patients with Type 2 diabetes in the ADVANCE trial. Health Qual Life Outcomes. 2007:5:21. doi:10.1186/1477-7525-5-21

30. Janssen MF, Lubetkin El, Sekhobo JP, Pickard AS. The use of the EQ5D preference-based health status measure in adults with Type 2 diabetes mellitus. Diab Med. 2011;28(4):395-413. doi:10.1111/j.14645491.2010.03136.x

31. Brooks R. EuroQol: The current state of play. Health Policy.1996; 37 (1):53-72. doi:10.1016/0168-8510(96)00822-6. [PubMed: 10158943]

32. Brazier J, Deverill M, Green C. A review of the use of health status measures in economic evaluation. J Health Serv Res Policy. 1999;4(3):174-84. [PubMed: 10538884].

33. Longworth L, Bryan S. An empirical comparison of EQ-5D and SF-6D in liver transplant patients. Health Econ. 2003;12(12):1061-7. doi:10.1002/hec.787. [PubMed: 14673814]. 
34. Dolan P, Gudex C, Kind P, Williams A. The time trade-off method: results from a general population study. Health Econ.1996;5(2):141-154. doi:10.1002/ (SICI)1099-1050(199603)5:2<141::AID-HEC189>3.0.CO;2-N

35. Li L, Fu A. Some methodological issues with the analysis of preferencebased EQ-5D index score. Health Serv Outcomes Res Methodol. 2009;9(3):162-176. doi:10.1007/s10742-009-0053-3

36. Huang IC, Frangakis C, Atkinson MJ, et al. Addressing ceiling effects in health status measures: A comparison of techniques applied to measures for people with HIV disease. Health Serv Res. 2008;43 (1 Pt 1):327-339. doi:10.1111/j.1475-6773.2007.00745.x

37. Pullenayegum EM, Tarride JE, Xie F, Goeree R, Gerstein HC, O'Reilly D. Analysis of health utility data when some subjects attain the upper bound of 1: Are Tobit and CLAD models appropriate? Value Health. 2010;13(4): 487-494. doi:10.1111/j.1524-4733.2010.00695.x

38. Lee WJ, Song KH, Noh JH, Choi YJ, Jo MW. Health-related quality of life using the EuroQol 5D questionnaire in Korean patients with type 2 diabetes. J Korean Med Sci. 2012;27(3):255-260. doi:10.3346/ jkms.2012.27.3.255

39. Solli O, Stavem K, Kristiansen IS. Health-related quality of life in diabetes: The associations of complications with EQ-5D scores. Health Qual Life Outcomes. 2010;8:18. doi:10.1186/1477-7525-8-18

40. Holmes J, McGill S, Kind P, Bottomley J, Gillam S, Murphy M. Health-related quality of life in type 2 diabetes (TARDIS-2). Value Health. 2000;3 (Suppl 1):47-51. doi:10.1046/j.1524-4733.2000.36028.x

41. Bagust A, Beale S. Modelling EuroQol health-related utility values for diabetic complications from CODE-2 data. Health Econ. 2005;14(3):217-230 doi:10.1002/hec.910
42. Sparring V, Nystrom L, Wahlstrom R, Jonsson PM, Ostman J, Burstrom K. Diabetes duration and health-related quality of life in individuals with onset of diabetes in the age group 15-34 years - aA Swedish population-based study using EQ-5D. BMC Public Health. 2013;13:377. doi:10.1186/14712458-13-377

43. Wexler DJ, Grant RW, Wittenberg E, et al. Correlates of health-related quality of life in type 2 diabetes. Diabetologia. 2006;49(7):1489-1497. doi:10.1007/ s00125-006-0249-9

44. Sakamaki H, Ikeda S, Ikegami N, et al. Measurement of HRQL using EQ-5D in patients with type 2 diabetes mellitus in Japan. Value Health. 2006;9(1): 47-53. doi:10.1111/j.1524-4733.2006.00080.x

45. Beaudet A, Clegg J, Thuresson PO, Lloyd A, McEwan P. Review of utility values for economic modeling in type 2 diabetes. Value Health. 2014;17(4):462-470. doi:10.1016/j.jval.2014.03.003

46. Greiner W, Claes C, Busschbach JJ, von der Schulenburg JM. Validating the EQ-5D with time trade off for the German population. Eur J Health Econ. 2005:6(2):124-130.

47. Badia X, Roset M, Herdman M, Kind P. A comparison of United Kingdom and Spanish general population time trade-off values for EQ-5D health states. Med Decis Making. 2001;21(1):7-16.

48. Johnson JA, Ohinmaa A, Murti B, Sintonen H, Coons SJ. Comparison of Finnish and U.S.-based visual analog scale valuations of the EQ-5D measure. Med Decision Making. 2000;20(3):281-289.

This work is licensed under a Creative Commons Attribution-NonCommercial 3.0 Unported License which allows users to read, copy, distribute and make derivative works for non-commercial purposes from the material, as long as the author of the original work is cited properly. 